Images du travail, travail des images

$5 \mid 2018$

Le travail à l'écran : mise en scène des groupes professionnels par les médias

\title{
La formation ecclésiale au prisme d'une série TV : dé-singularisation d'un dispositif
}

The formation of the priesthood through the prism of TV series : trivialisation of a training system

\section{Benoît Verdier}

\section{(2) OpenEdition}

\section{Journals}

Édition électronique

URL : http://journals.openedition.org/itti/816

DOI : $10.4000 /$ itti.816

Éditeur

Université de Poitiers

Référence électronique

Benoît Verdier, «La formation ecclésiale au prisme d'une série TV : dé-singularisation d'un dispositif », Images du travail, travail des images [En ligne], 5 | 2018, mis en ligne le 01 février 2018, consulté le 14 avril 2021. URL : http://journals.openedition.org/itti/816 ; DOI : https://doi.org/10.4000/itti.816

Ce document a été généré automatiquement le 14 avril 2021

Images du travail, travail des images 


\section{La formation ecclésiale au prisme d'une série TV : dé-singularisation d'un dispositif}

The formation of the priesthood through the prism of TV series : trivialisation of a training system

Benoît Verdier

1 Devenir prêtre au XXIe siècle interroge. Dans notre société marquée par la sécularisation, on assiste à " l'épuisement d'une identité qui a perdu son ancrage dans une culture commune, longtemps partagée au-delà du groupe amenuisé de ses fidèles " et qui amène le catholicisme à être socialement «illisible» (Hervieu-Léger, 2003). L'auteure utilise d'ailleurs le concept d'«exculturation » pour rendre compte de ce processus : phénomène de "dé-liaison ", de déconnexion entre la culture collective et la culture de l'église. Au-delà de cette disqualification culturelle, le déclin de l'institution catholique s'observe également tant dans la démographie des pratiquants réguliers que dans celle des prêtres ${ }^{1}$. En France, le nombre de ces derniers a été divisé par deux en vingt ans, passant de 29000 à 15000 aujourd'hui dont près de la moitié est âgée de plus de 75 ans. Depuis le début des années 1950, le renouvellement n'est plus assuré. Le nombre de candidats à la prêtrise n'a cessé de chuter : on comptait 4536 séminaristes en 1966, 1161 en 1980, ils n'étaient plus que 645 en 2014. Corrélativement le nombre d'ordinations passe de 646 en 1965 à 179 en 2014.

2 Notre contribution s'intéresse à ce groupe professionnel particulier - les clercs de l'Église catholique romaine - qui, comme le rappelle Jean-Paul Willaime dans une réflexion sur les types historiques des experts religieux, sont «des spécialistes d'un domaine particulier de connaissances et d'activités : le domaine du divin, [...] habilités à répondre à une clientèle par des prestations d'un type déterminé [...] sur la base de la relation interpersonnelle et qui de façon extrêmement diverse et avec des légitimations différentes, s'occupent de Lebensführung, de conduite de la vie » (1986, 30-31). Ils sont à la fois des agents d'une Institution légitime qui leur délègue une autorité et des 
experts de savoirs revendiqués dans "le champ de la manipulation symbolique " (Bourdieu, 1985, 255).

3 Instances de socialisation, les séminaires sont les lieux où l'Institution organise ce que nous dénommerons par la suite la formation ecclésiale des futurs prélats. Outre les indications du concile Vatican II (Optatam Totius), cette formation est régie par deux textes majeurs : l'exhortation apostolique de Jean-Paul II Pastores dabo vobis et la ratio des séminaires, votées par les évêques de France en 1997 (Le Stang, 2008, 355). Très brièvement, le dispositif s'étale sur six années et s'organise en deux cycles d'enseignement pendant lesquels les séminaristes approfondissent la philosophie, la théologie mais également le droit canon, la liturgie, l'exégèse, etc. Comme le rappelle Gérard Le Stang, cette formation se structure en quatre piliers. Le premier, le pôle de la formation humaine regroupe tout ce qui a trait à «la connaissance de soi, la vie communautaire, la formation de la vie affective et au célibat... ». Le second correspond au pôle de la vie spirituelle qui comprend «la prière, les retraites, la liturgie des heures, la vie sacramentelle, l'accompagnement spirituel... ». Le troisième concentre les études («souvent réalisées dans le cadre d'Instituts ou Facultés de théologie ouverts aux laïcs non séminaristes »). Et enfin le dernier, le pôle de la formation pastorale qui rassemble "les expériences apostoliques, paroissiales et leurs relectures, finalise l'ensemble. Ces axes de formation sont mis en œuvre dans le cadre d'une vie communautaire avec un suivi personnalisé » (Ibid., 355).

Dans cet article, nous souhaitons investiguer la représentation de cette formation proposée par la série Ainsi soient-ils ${ }^{2}$ qui situe son action dans un séminaire de l'Église catholique à Paris. Cette fiction suit le développement personnel et professionnel de cinq jeunes adultes qui, tout au long des deux premières saisons, vont "apprendre " leur futur métier de prêtre, qu'ils exerceront dans la troisième. Comment une série télévisée ayant pour objet une religion, le «croire», peut-elle investir la professionnalisation de ces fonctionnaires du culte? Quels sont les lieux et les actions de formation privilégiés dans la série? Quelles sont les représentations des dispositifs de formation ? Quels professionnels en devenir la série donne-t-elle à voir ?

Pour apporter des éléments de réponse à ces questionnements, nous procèderons en trois temps après avoir brièvement présenté la série. Tout d'abord, nous exposerons les éléments de monstration de cette formation qui tendent à la dé-singulariser pour ensuite s'attarder sur les modalités de formation et les contre-stéréotypes proposés. Enfin, nous discuterons ces différents résultats à l'aune du discours médiatique de l'Église catholique romaine française.

\section{La série Ainsi soient-ils}

6 Créée par David Elkaïm, Bruno Nahon, Vincent Poymiro et Rodolphe Tissot, et produite par Zadig productions et Arte France, la série situe l'action dans un séminaire de l'Église catholique en plein cœur de Paris dans le quartier latin. Elle suit l'arrivée, la vie et la transformation de cinq jeunes hommes issus de milieux sociaux différents et aux parcours et motivations très diverses. Ce sont cinq jeunes bien ancrés dans leur époque (contexte du XXIe siècle en France) que tout oppose mais que la foi rapproche.

7 Le choix de l'institution religieuse, l'Église catholique, n'est pas si anodin. En effet, depuis de nombreuses années, l'Église catholique est sous le feu des médias par toutes les affaires qui la concernent: pédophilie, mariage des prêtres, secret du Vatican, 
Banque du Vatican... Selon les auteurs, il ne s'agit pas de faire un pamphlet contre l'Église catholique, mais bien de «créer des personnages et les ancrer dans la réalité, dans le monde, dans la société contemporaine $»^{3}$. D'ailleurs, dans le dossier de presse et les interviews des scénaristes et/ou du producteur, il est clairement affiché que la série ne se destine pas uniquement aux catholiques (pratiquants ou non). Elle s'adresse à tous: croyants ou non. On retrouve dans cette promesse de la série le caractère fédérateur des produits culturels télévisuels.

Le corpus étudié pour cette communication se compose des 16 épisodes de 52 minutes des saisons 1 et 2 . Nous avons privilégié une analyse au croisement de l'analyse sémionarrative et de contenu (Jost, 2007; Esquenazzi, 2010) et notre travail s'inscrit notamment dans la lignée de ceux de Sabine Chalvon-Demersay (notamment 1999, $2000,2005,2011)$ qui sondent les fictions pour investiguer le social. Il s'agit, dans cet article, de questionner la représentation de la formation ecclésiale à travers le prisme médiatique d'une série télévisée ou, dit autrement, d'analyser son avatar ${ }^{4}$ médiatique pour reprendre le terme utilisé par Macé $(2001,2002)$ - forme particulière de représentation de la « réalité » du social. À l'instar d'Éric Macé, l'auteure nous propose de les déplier, c'est-à-dire : «de cartographier leurs points de vue, de les mettre en perspective, de les documenter à la fois sur le plan technique et sur le plan politique, de prendre au sérieux les idées qu'ils développent » (Chalvon-Demersay, 2015, 38).

Dans cet article, nous nous attachons donc à scruter la sémiotisation de cette formation enchâssée dans son « drame social du travail »

\section{De la trivialité de la formation ecclésiale}

La trame narrative réside dans la professionnalisation de séminaristes. L'univers de la formation ecclésiale est au cœur de la série et parce qu'ils « se recouvrent et s'appellent mutuellement » (Le Stang, 2008, 355), les quatre pôles (présentés précédemment et qui la structurent) s'imbriquent et participent du récit. Se trouvent (r)assemblés et figurés tous les thèmes qui jalonnent la vie d'une formation classique: du recrutement jusqu'aux soirées estudiantines, en passant par la correction des copies et leur rendu, le travail personnel (en bibliothèque ou dans sa chambre), la gouvernance et un audit de la structure... Tout y est, mais certains termes diffèrent. Par exemple, on ne parle pas d'un oral pour un examen particulier mais d'un concours d'homélie (Père Cheminade, S2E56), point de journée portes ouvertes mais journée des vocations et les stages sont qualifiés « d'insertion pastorale ». Tous les lieux sont investis : la classe, l'amphithéâtre, le self, la salle de repos ou le bureau des étudiants, la bibliothèque, la salle du conseil, la chambre de l'étudiant (internat), la cours (le patio du séminaire), les couloirs...). 
Image 1. Déjeuner au self

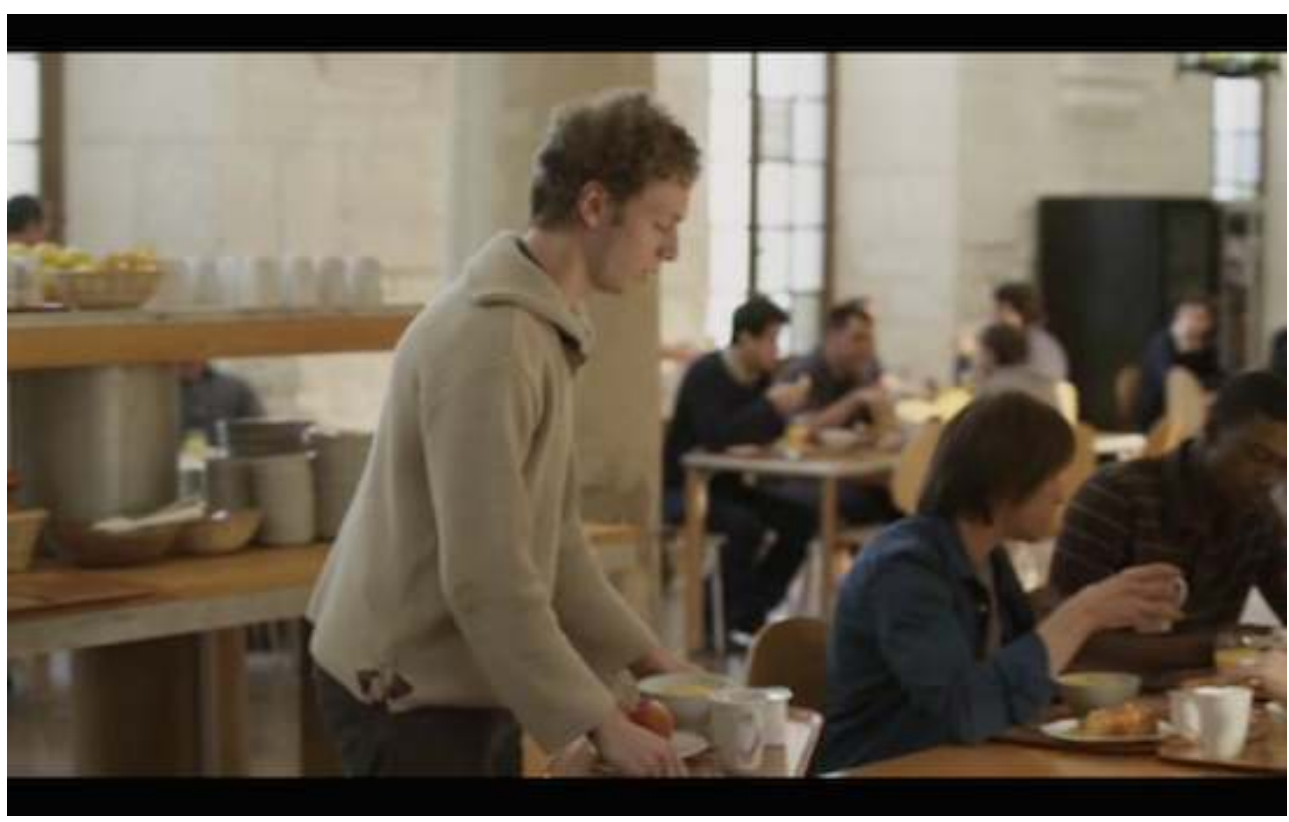

Capture d'image de la série Ainsi soient-ils (S1E1)

(c) Zadig Productions - Arte

Image 2. Conseil de la formation

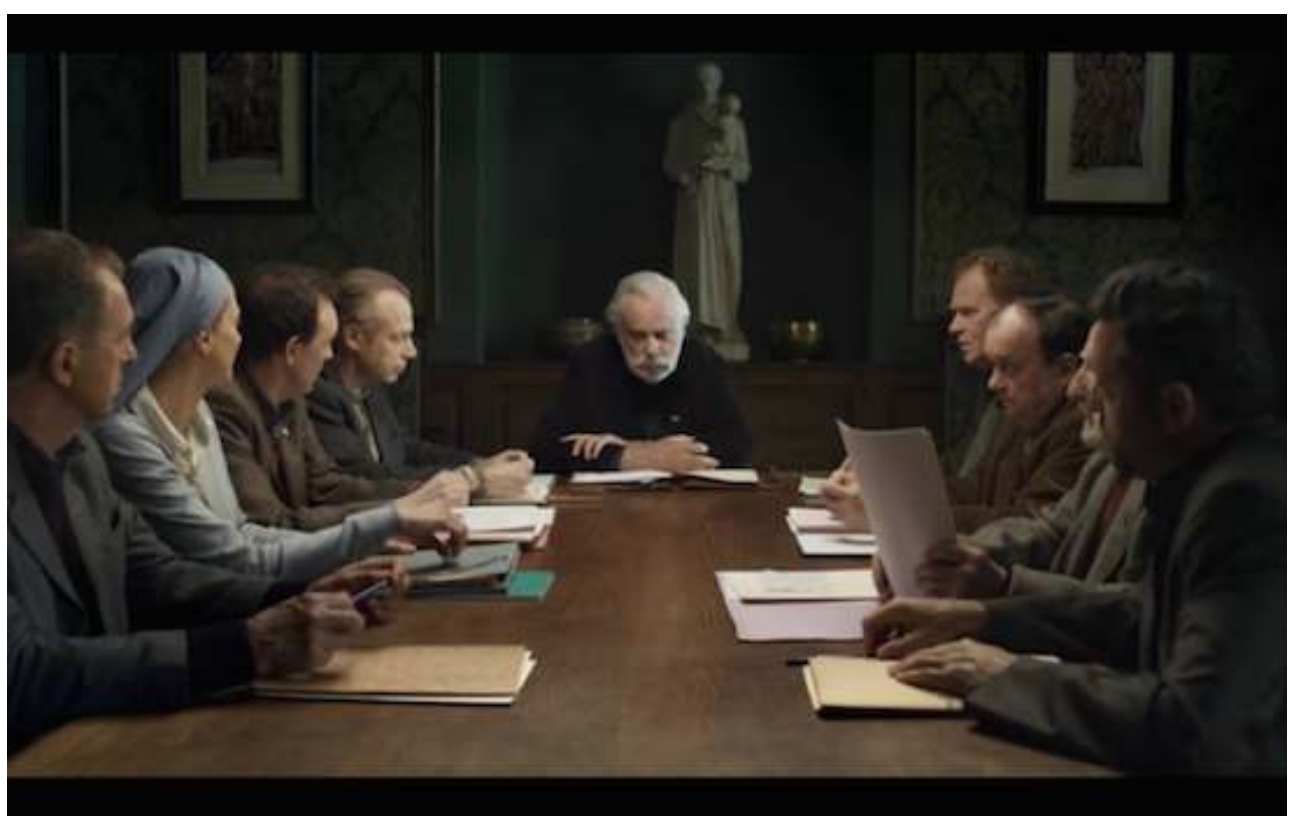

Capture d'image de la série Ainsi soient-ils (S1E5)

(c) Zadig Productions - Arte

11 La temporalité de la série respecte le calendrier social d'une part et liturgique d'autre part. Les diverses fêtes liées à la culture chrétienne (de la Toussaint à Pâques en passant par Noël) ponctuent chaque saison. D'ailleurs, les séries se basent souvent sur ces événements qui rythment la vie de leurs téléspectateurs (comme la traditionnelle fête de Thanksgiving dans les séries américaines). En outre, telle une formation post-bac, les deux premières saisons suivent le calendrier universitaire (de septembre à juin). La 
rentrée est donc l'occasion de présenter l'ensemble choral des personnages principaux lors du premier épisode de la saison 1 permettant ainsi de proposer la thématique du recrutement et de s'interroger sur la question de la vocation à ce métier relativement singulier.

Ensuite, vient l'intégration des premières années (le «bizutage »). C'est l'occasion de la première fête « estudiantine » (S1E1). Les épisodes suivants placent les séminaristes en cours, à la bibliothèque mais également dans des cours "extra-muros" dans une université parisienne (S1E3).

13 La vie estudiantine est célébrée : les cours, les discussions, la musique, les amours, etc. D'ailleurs, lors d'un conseil du séminaire (S1E6), l'un des points à l'ordre du jour concerne le bureau des séminaristes (équivalent du Bureau des étudiants, BDE) et « sa demande traditionnelle de carnaval ». L'échange entre les pères est intéressant : pour certains, il s'agit d'« une tradition : les séminaristes ont le droit à leur folklore » (S1E6) alors que d'autres, hostiles à cette initiative, dénoncent "la perpétuation de ces chahuts comme une vulgaire école de commerce » (S1E6). Dans la série, de nombreuses soirées ponctuent donc l'année des séminaristes comme cela se fait dans toute formation.

Tout au long des épisodes, par les lieux, les thèmes abordés, la temporalité choisie, les séminaristes évoluent dans un monde en tout point pareil à n'importe quelle formation. La formation ecclésiale représentée dans cette série n'est donc pas énigmatique tant pour un public non-catholique (telle que la promesse nous l'indique) que pour un auditoire plutôt cultivé et éduqué auquel s'adresse la chaîne Arte. Socialisé par une formation supérieure post-bac, ce public en connaît le fonctionnement et les institutions. Alors que la fête des séminaristes bat son plein (soirée d'intronisation des nouveaux), un échange entre le père supérieur du séminaire des Capucins et une sœur est signifiant :

«Ma sœur, n'oubliez pas que nous sommes à la lisière du quartier latin. Les premiers chahuts des étudiants ont eu lieu aux alentours de 1450. Ils étaient très violents, contestataires, licencieux et ils étaient tous des étudiants en théologie » (S1E1).

En rappelant que les universités avant d'être républicaines et laïques étaient des universités de théologie détenues par le clergé, la série replace ce dispositif de formation dans l'histoire de notre société et de notre rapport au savoir.

La sémiotisation de la formation ecclésiale réalisée tend vers une confusion des genres tant elle se présente en parallèle et surtout dans un mode analogue à une formation classique, universitaire de surcroît, réalisée dans un mode authentifiant (Jost, 2007). Le téléspectateur ne se sent pas égaré dans la mesure où s'opère une banalisation du lieu et du dispositif. Dès la première saison, il s'agit de positionner ces séminaristes comme des jeunes de leur époque dans un enseignement semblable à celui du supérieur classique. L'objectif semble être de dé-singulariser cette formation.

\section{Entre bricolage et formation expérientielle}

17 Plusieurs modalités de formation se donnent à voir dans la série. D'une part, les cours à effectif restreint se déroulent toujours dans une petite salle au sein du séminaire pour des disciplines comme le grec ancien, la patristique, etc. Les enseignants sont des pères. D'autre part, les cours donnés face à un effectif plus conséquent en amphithéâtre - 
exclusivement - se tiennent ailleurs qu'au séminaire, soit dans les locaux de la Conférence des évêques de France (CEF, lieu de la gouvernance ecclésiale) soit dans une université parisienne publique par définition laïque. À l'université, c'est un cours de philosophie donné par un enseignant ; à la CEF, il s'agit d'une formation professionnelle à la communication pour de futurs clercs mise en œuvre par un prêtre (le père Soubiran) lui-même chargé de communication de cette organisation. Dans ce cas, l'ensemble du dialogue se fait dans une semi-pénombre avec la vidéoprojection de publicités du denier du culte réalisées par le communiquant.

Image 3. "Cours au séminaire »

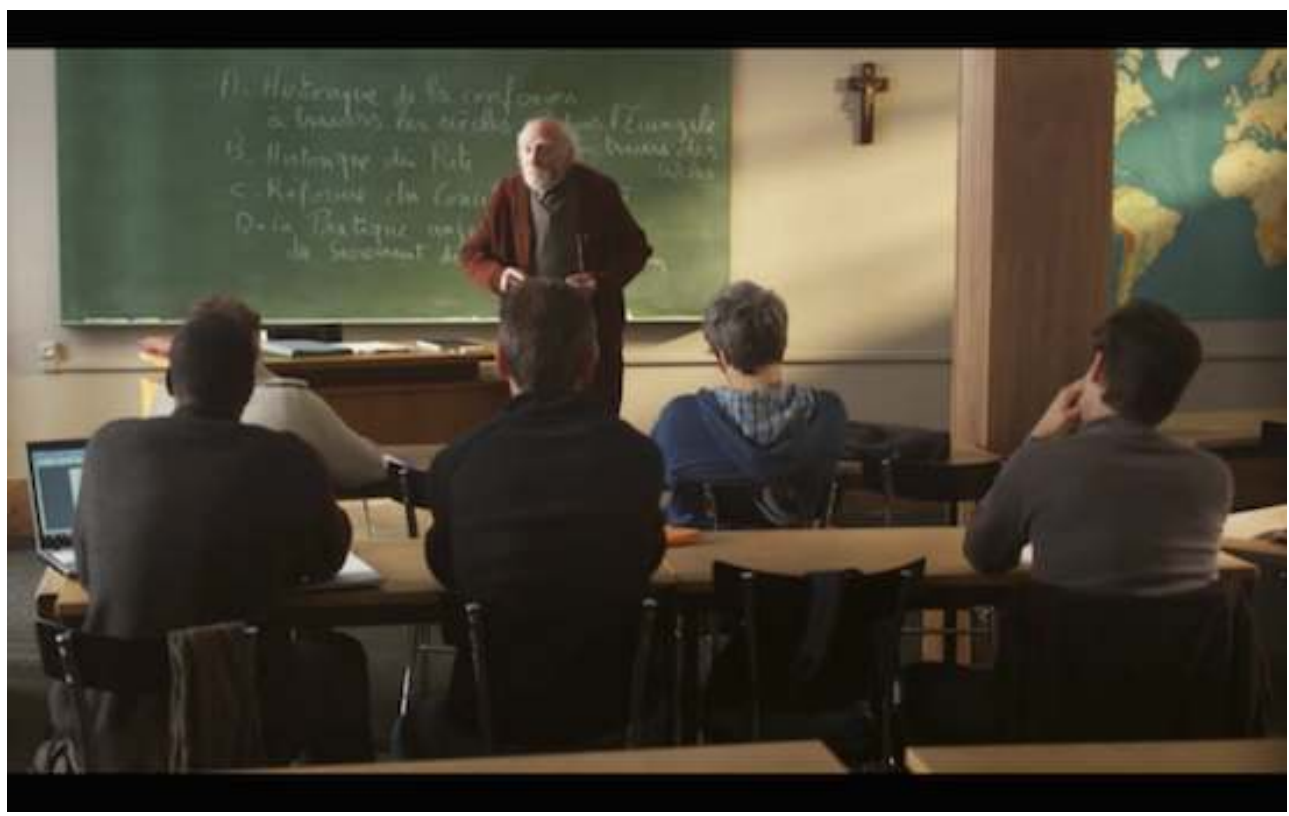

Capture d'image de la série Ainsi soient-ils (S1E3)

(c) Zadig Productions - Arte 
Image 4. "Cours en amphi »

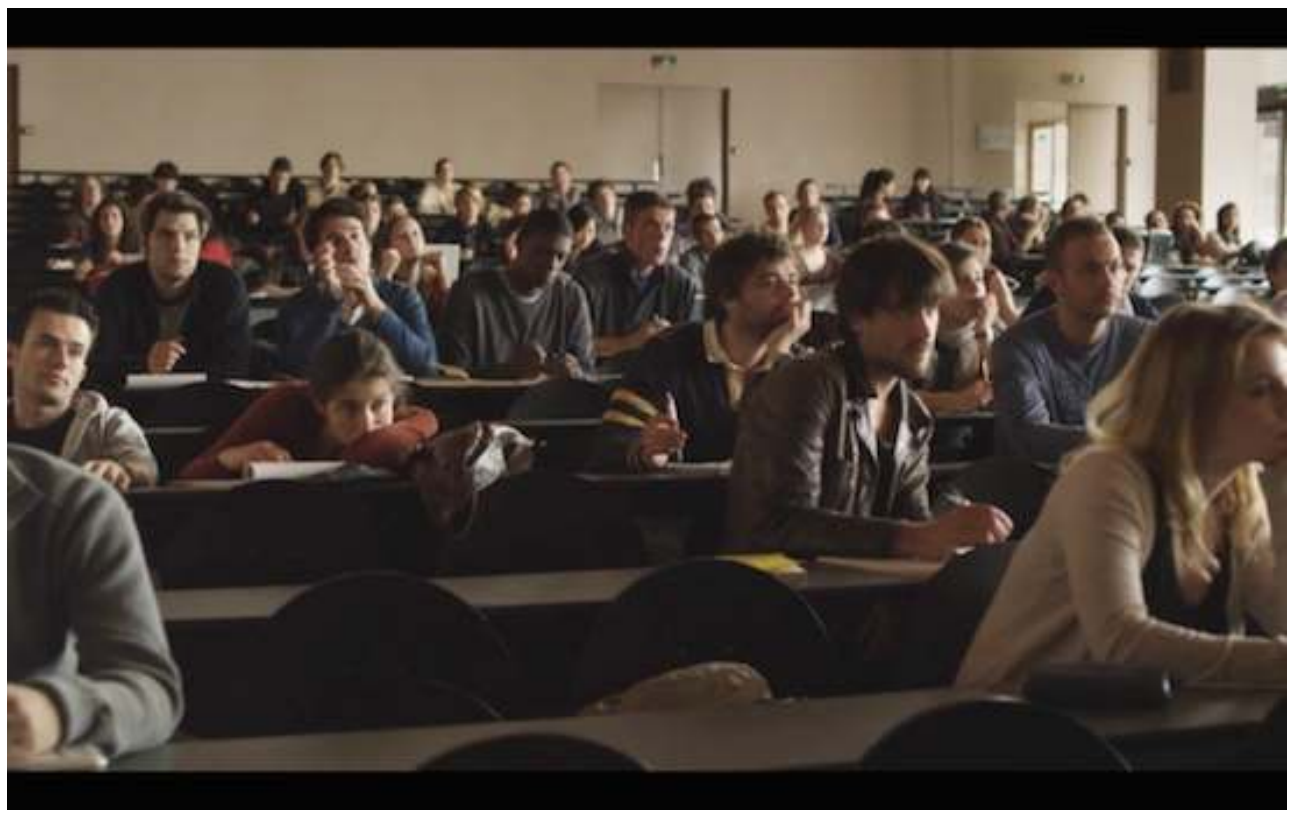

Capture d'image de la série Ainsi soient-ils (S1E3)

(c) Zadig Productions - Arte

18 En amphithéâtre, le professeur de philosophie propose de dialoguer avec son public et interroge directement un individu :

«Maintenant j'aimerais que vous preniez la parole, que vous exprimiez votre opinion sur le programme de cette année qui est donc la question libertaire! Vous? Jeune homme ? Qu'est-ce que vous avez à nous dire, on vous écoute » (S1E3).

Il en va de même lors la formation à la CEF, où le père Soubiran introduit son propos par ces mots :

"Alors, à chaque image que je vais vous proposer, j'aimerais que vous lanciez, comme ça, à chaud, vos réactions, comme ça, sans inhibition » (S2E3).

Invités à donner leur opinion, les apprenants n'hésitent pas et expriment leur avis (négatif), voire leur désaccord. Dans les deux cas, cette réponse des étudiants provoque un sentiment de malaise de la part de l'enseignant en amphithéâtre :

À l'université : «S'il vous plait, c'est un cours de philo ici, ce n'est pas le café du commerce!»(S1E3);

À la CEF : «Je vous ai demandé d'être franc, donc, je ne vais pas vous le reprocher. Maintenant, si vous êtes ici c'est, a priori, pour apprendre quelque chose et ne pas donner des leçons avec un petit sourire en coin. C'est le but d'un stage, d'être ouvert, curieux, désireux d'acquérir de nouvelles connaissances, sur la base de l'expérience de vos aînés » (S2E3).

Cette modalité de formation (interactivité enseignant/étudiant) donnée à voir ici se présente comme un moment d'échange qui tourne très vite au désavantage de celui qui le propose (pourtant censé le maîtriser) : l'enseignant/formateur. D'un point de vue fictionnel, «il s'agit avant tout de trouver dans ces lieux des ressorts narratifs qui permettent de mettre les personnages dans des situations qui les fassent évoluer psychologiquement $»^{7}$ soulignent les scénaristes de la série, Vincent Poymiro et David Elkaïm. Et celle-ci en représente un. Néanmoins, on voit poindre la satire sociale lors du 
dialogue entre deux des séminaristes s'installant sur les bancs de l'amphithéâtre d'une faculté parisienne :

Raphaël : « Eh bien, il nous a gâtés Fromenger avec ses cours extra-muros. T’as vu la gueule de la fac? !

Guillaume : « C'est sûr que ça doit te changer de sciences po ! (S1E3).

l'enfermement et l'imprégnation des textes anciens, loin des réalités du monde extérieur. L'utilisation de ces cours extra-muros dans la narration n'est pas tant pour coller au point de vue d'une part, authentifiant de la formation ${ }^{8,}$ qu'à celui, d'autre part, du prêtre ouvrier de gauche représentant le père supérieur. L'habitus professionnel qui pourrait se décliner ici en habitus sacerdotal semble s'acquérir par un savant mélange de travail individuel sur les textes, d'expérience individuelle et collective en se frottant à la réalité du monde extérieur lors de stages «d'insertion pastorale " (tels que l'Institution les nomme). José, l'un des séminaristes, précise aux étudiants de l'amphithéâtre, manipulés et indisposés par leur présence :

«nous sommes venus ici justement pour ne pas nous exclure du monde, de sa réalité, pour être à son écoute, pour nous laisser envahir par elle. Qu'est-ce qui va nous arriver si la réalité se retire dès que nous tentons de l'approcher? Nous aussi, nous sommes le monde avec nos erreurs, nos peurs »(S1E3).

26

Et la série regorge d'exemples : les stages dans différentes associations, les actions des séminaristes dans une paroisse à Noël ou auprès d'un groupe de sans-papiers dans un squat, etc. sont autant de lieux de formation pour les séminaristes. 
27 Le parangon de la formation présentée dans cette fiction sérielle demeure celui privilégiant l'expérience. Alors que nous sommes censés rester dans un séminaire et voir un dispositif classique et très organisé, c'est tout un ensemble hétéroclite de modalités de formation qui est proposé. En jouant sur ce contre-stéréotype (Macé, 2007), le cadre formatif strict (clos) du séminaire se transforme en autoformation ouverte sur le monde -, où toute expérience de vie prend une dimension formative. Du point de vue de l'Institution, le passage à l'université demeure un moyen d'aborder différemment les (ou d'autres) textes, l'enseignement, la matière - la philosophie -, mais également d'acquérir une (nouvelle) expérience en se frottant au monde " réel ", auprès d'étudiant·e·s de leur âge. En effet, la thématique de la " maturité affective » se trouve ainsi abordée questionnant la chasteté (voire plus globalement les rapports homme/femme), l'homosexualité. Ici, l'expérience formative, en termes de ressorts narratifs, revêt tel Janus une double dimension. Elle peut être à la fois jubilatoire, aventureuse et pleine de promesses mais aussi emplie de contraintes, de responsabilités et de déceptions (sinon d'échecs).

En contrepoint, cette série convoque le modèle du bricolage et l'art d'improviser. Les séminaristes sont livrés à eux-mêmes, aucun retour réflexif individuel quant à la formation n'est proposé. La formation par l'expérience semble se suffire à elle-même, figurée comme intrinsèquement "formative " sans nul besoin d'une réflexivité. Dans cette série, cette réflexion introspective s'établit d'une manière collective et toujours en lien avec l'expérience de vie des membres de la communauté par l'intermédiaire de l'homélie, sermon réalisé au cours de l'office (Verdier, 2016). En effet, chaque épisode propose cette modalité narrative, «composée d'éléments discursifs atemporels favorisant l'anonymat et donc l'individualisation dans un nous collectif », comme une synecdoque de l'introspection (Ibid).

La représentation de la formation proposée ici se développe donc à travers des contrestéréotypes: ouverture sur le monde privilégiant les relations interpersonnelles et expériences individuelles. La focale semble donc se situer sur l'acquisition de compétences liées à une pratique sur le terrain: animation, gestion de structure, d'équipe. Se trouve ainsi sémiotisée la professionnalisation de la fonction chef d'orchestre du prêtre telle que la nomme Céline Béraud (2006). «Dans un contexte d'approfondissement et de complexification de la division du travail religieux, le prêtre tel un cadre au sein de l'institution qu'est l'Église locale, préside, organise, et anime les communautés qui sont sous sa responsabilité » (Ibid., 86).

\section{Comparaison avec deux documentaires officiels de promotion de la vocation}

30 Les médias opèrent une sélection et un cadrage des informations. Pour les collectifs de travail (et par extension les institutions qu'ils représentent) régulièrement projetés sur le devant de la scène médiatique, il leur faut accepter le processus de "stéréotypage » engendré par les contraintes et les routines en vigueur dans la fabrication des fictions (Voirol, 2005a, 107). Ce ne fût pas le cas lors de la diffusion de la première saison qui a suscité une controverse médiatique importante alimentée par un discours anti-série de la part de nombreux acteurs de l'Église. Aussi, il convient maintenant de faire dialoguer les différents résultats à l'aune du «discours officiel » de l'Église catholique romaine 
ou, dit autrement, de mettre en regard la série avec la mise en image de la formation ecclésiale par ses propres acteurs.

Même si notre contribution ne se concentre pas sur ce matériau, nous avons analysé deux films documentaires ${ }^{9}$ dont l'objectif est la promotion de la vocation. Le premier $\mathrm{s}^{\prime}$ intitule Devenir Prêtre. Il est produit par la chaine $\mathrm{KTO}^{10}$ et réalisé par Dominique Féret en 2010. Le synopsis du documentaire se rapproche de la série : «Peut-on apprendre à devenir prêtre comme on apprend un métier? Tourné au séminaire des Carmes, sur une période de plusieurs mois, ce film s'attache à suivre leur itinéraire et nous fait pénétrer dans un lieu et une formation peu connus du grand public ». Le second, Prêt(re) pour se donner, est réalisé par Véronique Brechot et produit par le séminaire de Lille en 2012. Là encore, la proximité avec la série est édifiante : « la vie au séminaire a souvent été une source romanesque exploitée par la littérature et le cinéma. Mais pour le grand public, c'est une histoire du passé, un peu étrange, et pour les communautés catholiques, qui vivent durement la chute des vocations, une source d'interrogations. Il souhaite montrer une image plus dynamique et positive de la vie au séminaire, et de ces jeunes gens qui passent quelques années en vue de se consacrer à la prêtrise ». Tout comme dans la série, la réalisatrice a choisi 5 séminaristes pour incarner différents profils.

La formation ecclésiale figurée dans ces vidéos de promotion propose des cours au séminaire en petit effectif, en amphithéâtre à la faculté de théologie, des exposés, des soirées, des séminaristes en stage (en paroisse ou "d'insertion pastorale " selon la dénomination de l'Institution), l'apprentissage des rituels du culte et de leurs gestes associés... On retrouve une indiscutable similitude dans les scènes jouées dans la série et celles filmées dans les documentaires: parfois la proximité est telle qu'elles pourraient se substituer. Ces deux œuvres audiovisuelles montrent donc les mêmes thématiques et sensiblement le même traitement de la formation que dans la série.

Lors de la diffusion de la première saison, l'Institution a réagi dans les médias par des propos négatifs parfois très virulents à son égard en arguant d'une mise en fiction de la formation ecclésiale très éloignée de la réalité :

"Quel curieux lieu que ce "séminaire des capucins" où les candidats au sacerdoce n'ont ni cours, ni exposés, ni examens, ni mémoires à rendre, ni recherches en bibliothèque ! $»^{11}$.

Pourtant, comme nous l'avons vu, la série ne cesse de présenter la formation de ses clercs en train de se faire. Les séminaristes sont en cours, en stage, en travail individuel à la bibliothèque... et rendent des copies, participent à un concours d'homélie... Tout comme les séries qui traitent de l'éducation au sens large (l'Instit, Madame le proviseur ou dans un format plus court Pep's), les cours sont en partie évidés de leur contenu pour se polariser sur les interactions entre les personnages et leurs évolutions psychologiques (Chalvon-Demersay, 2011). En termes de décor et de trame narrative, le « drame social du travail » (Hughes, 1996) organise donc cette fiction sérielle. Tout comme il structure également les documentaires réalisés pour et par l'Institution ecclésiale. 
Image 5. "Travail en bibliothèque »

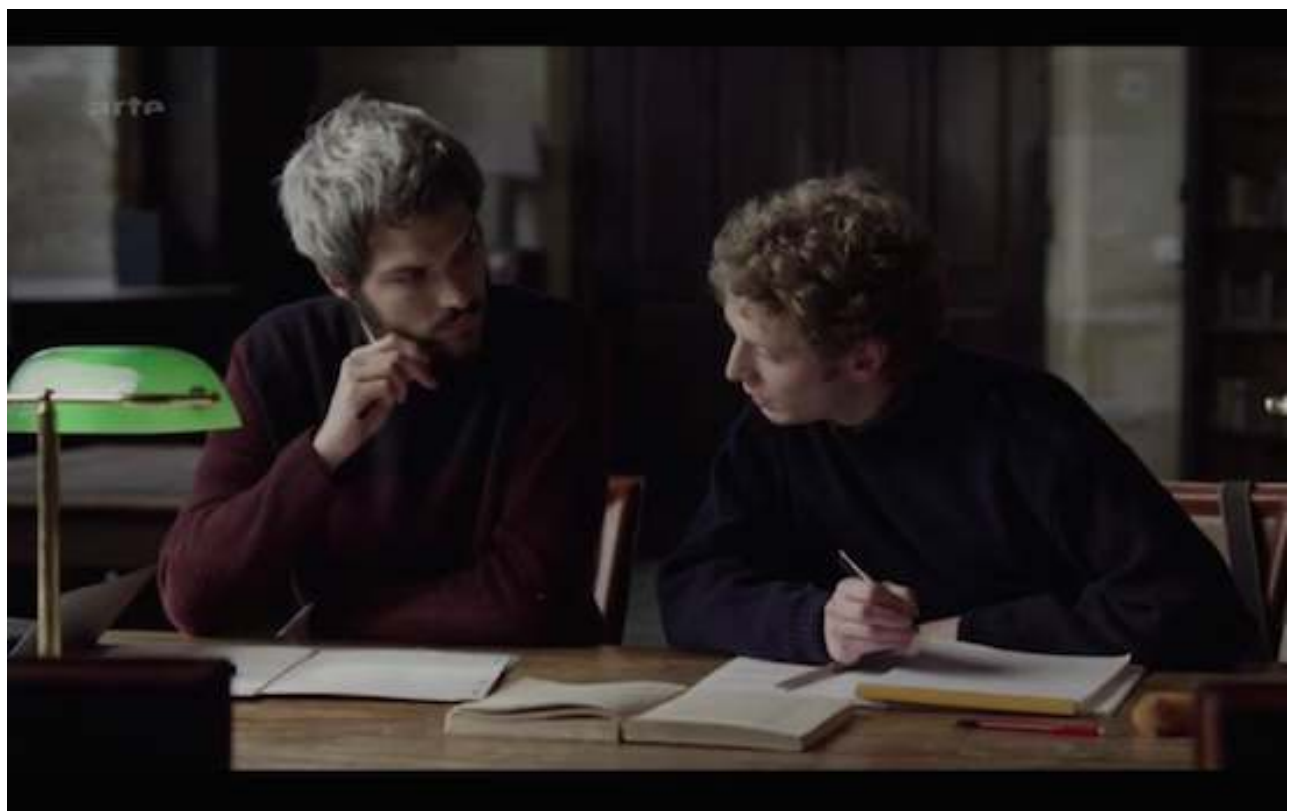

Capture d'image de la série Ainsi soient-ils (S2E3)

(C) Zadig Productions - Arte

Image 6. « Travail individuel dans sa chambre »

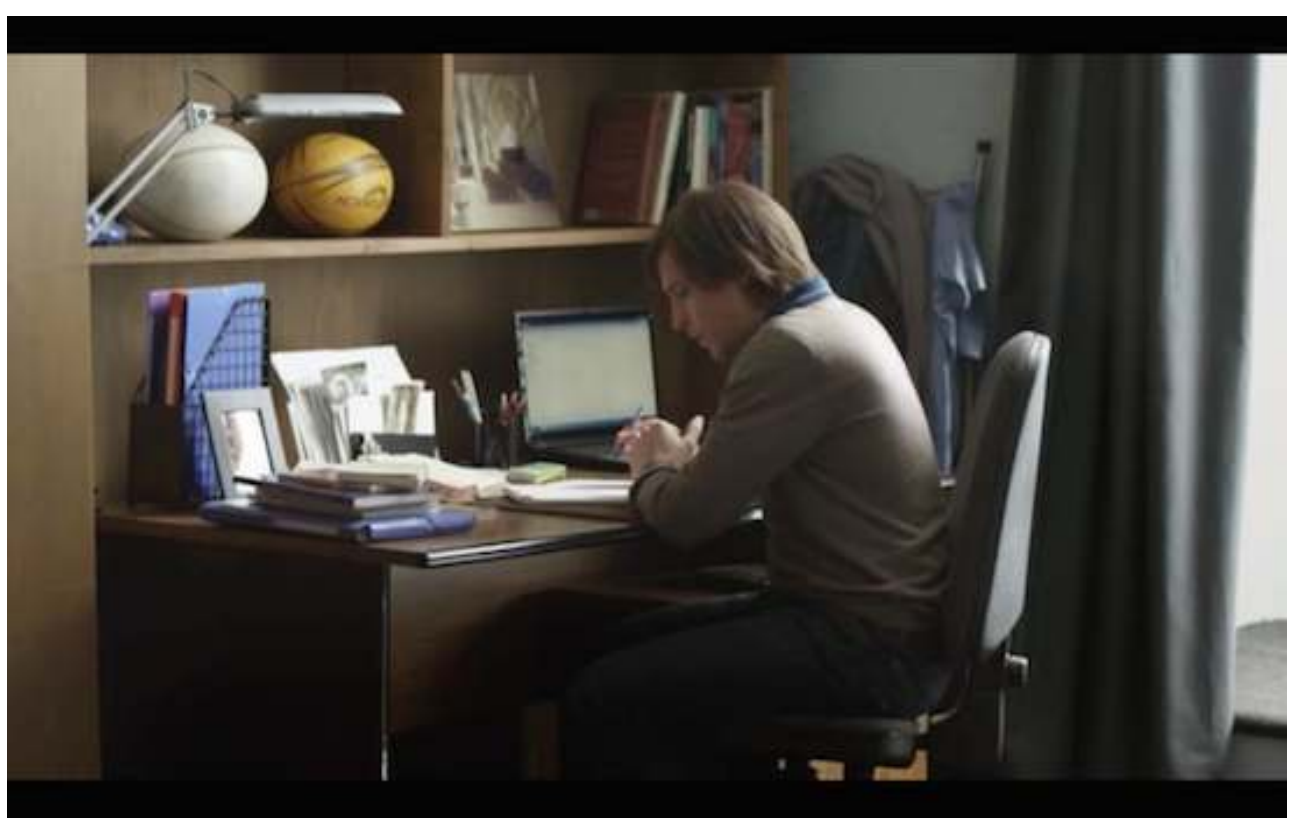

Capture d'image de la série Ainsi soient-ils (S1E3)

(c) Zadig Productions - Arte 
Image 7. «Rendu de copies»

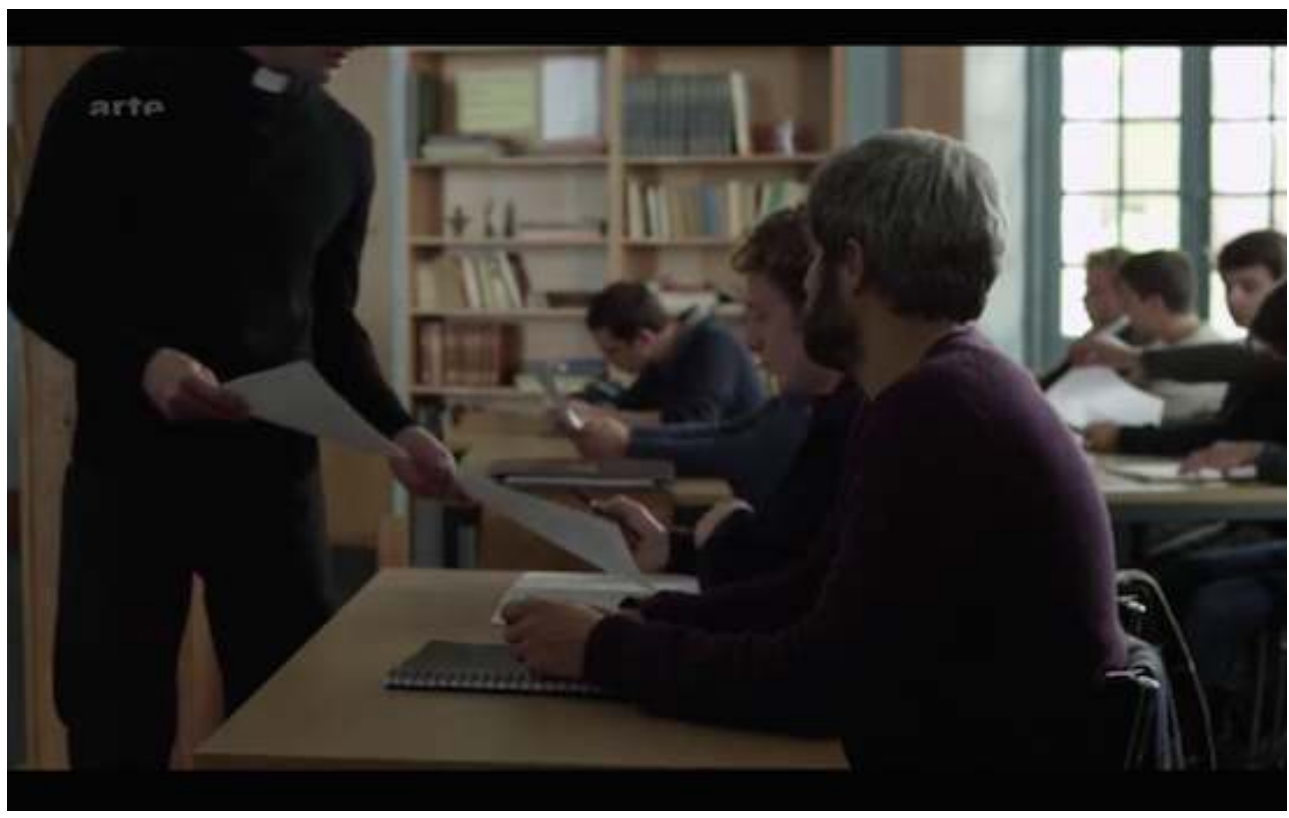

Capture d'image de la série Ainsi soient-ils (S2E3)

(c) Zadig Productions - Arte

Au regard de la dé-singularisation opérée dans la série, la figure du fonctionnaire du culte - médiateur entre le sacré et le profane - s'enracine plutôt dans le registre professionnel qui relève d'une légitimation techno-bureaucratique. Il s'agit ici de montrer qu'on forme avant tout des professionnels, et des professionnels de la médiation humaine spécialisés dans le "champ de la manipulation symbolique " (Bourdieu, 1985). Déjà investie par les prêtres contestataires des années 1960-1970, cette catégorie l'est encore « dans les discours des jeunes clercs, par la mobilisation du registre de la compétence, voire un attrait pour les techniques managériales issues du monde de l'entreprise " (Béraud, 2009, 186). Et, nous la retrouvons dans les vidéos de promotion de la vocation réalisées par l'Institution. Ainsi, un des points de tension constitutifs de la visibilité médiatique définie comme "un horizon de sens et de catégorisation conflictuel où se confrontent continuellement des définitions contradictoires de "ce qui vaut d'être vu" " (Voirol, 2005b, 19) entre l'Institution et la série ne se niche donc pas au niveau de ce régime de monstration de la formation ecclésiale.

\section{Conclusion}

La série Ainsi soient-ils s'inscrit dans et réfléchit la société contemporaine du XXIe siècle (Verdier, 2014). En se référant aux travaux de Touraine, Beck et Giddens, Jean-Paul Willaime propose le terme d'ultramodernité pour nommer la société dans laquelle nous vivons. Pour l'auteur, «c'est toujours la modernité, mais la modernité désenchantée, problématisée, autorelativisée. Une modernité qui subit le contrecoup de la réflexivité systématique qu'elle a enclenchée » $(2008,18)$. En proposant notamment une formation ecclésiale dé-singularisée, la série opère un processus de "profanation" au sens d'Agamben (2005) du dispositif de formation. Pour l'auteur, tandis que les choses consacrées "se voyaient soustraites au libre usage et au commerce des hommes » et 
reposaient ainsi sur une «indisponibilité spéciale qui les réservait aux dieux du ciel ", la profanation suppose une dynamique contraire et consiste en une sortie de la sphère du sacré et « une restitution au libre usage des hommes » $(2005,95)$. Profaner, engager des déplacements "ne signifie pas seulement abolir et effacer les séparations mais apprendre à en faire un nouvel usage, à jouer avec elles » $(2005,115)$. Ce jeu semble se révéler dans cette fiction au travers de la représentation des dispositifs de formation tant ecclésiale qu'universitaire. En effectuant une opération de dé-singularisation d'une formation éprouvée par ses représentants comme spécifique et particulière, il y a déplacement entre deux espaces distincts : du sacré vers le profane, de l'exceptionnel vers le trivial.

37 Cette série propose une formation ecclésiale banalisée sans cesse repositionnée dans le champ d'une formation classique du supérieur. Elle n'a de cesse de dépersonnaliser cette formation " particulière » à un métier " particulier » en la renvoyant à un modèle de formation trivial, dépossédant alors les clercs d'une caractéristique différenciante (pour ne pas dire discriminante). Ces jeunes séminaristes sont présentés comme des étudiants lambda dans une formation lambda - ouverte sur le monde - qui acquièrent un savoir (ici théorique sur les textes de l'Église) mais également des compétences (ici en relations humaines) dans un environnement privilégiant l'expérience.

Cette opération de dé-singularisation de cette formation pourrait être pensée comme une forme supplémentaire de sécularisation. Or, comme nous l'avons vu, cette représentation n'est pas éloignée de celle proposée par l'Institution ecclésiale dans ses vidéos de promotion de la vocation. L'argumentaire médiatique de l'église sur l'inexacte représentation du dispositif de formation des professionnels du culte dans la série ne tient donc pas. Comme le montrent les recherches de Céline Béraud (2006, 2007, 2009), professionnalisation et néosacerdotalisation de la prêtrise coexistent dans les dispositifs de formation mis en place par l'Institution ecclésiale et «constituent deux symptômes mais également deux tentatives pour pallier la légitimité désormais problématique des prêtres » $(2009,196)$. Comme la première catégorie est commune à la série et au discours officiel de l'Église, il faudrait s'attarder sur la seconde et interroger la sémiotisation de la réactivation de l'idéal vocationnel sacerdotal proposée par la série pour la comparer avec celle de l'Église dans ses vidéos de promotion. C'est peut-être là que se cristallise secrètement la contestation.

\section{BIBLIOGRAPHIE}

Agamben G. (2005), Profanation, Paris, Rivages.

Beck U. (2001), La société du risque. Sur la voie d'une autre modernité, Paris, Aubier.

Béraud C. (2006), Le métier de prêtre, Paris, Éd. de l'Atelier.

Béraud C. (2009), « Mutations de la formation pour le clergé catholique : entre idéal sacerdotal et valorisation de la compétence », in Demazière D. et Gadéa C. (dir.), Sociologie des groupes

professionnels. Acquis récents et nouveaux défis, Paris, La Découverte, p. 186-196.

Images du travail, travail des images, 5 | 2018 
Bourdieu P. (1985), « Le champ religieux dans le champ de manipulation symbolique », postface, Les nouveaux clercs: Prêtres, pasteurs et spécialistes des relations humaines et de la santé, Genève, Labor et Fides, p. 255-261.

Chalvon-Demersay, S. (1999), «La confusion des conditions. Une enquête sur la série télévisée Urgences ", Réseaux, nº 95, vol. 17, p. 235-283.

Chalvon-Demersay S. (2000), « Pour une approche sociologique des œuvres de fiction télévisée », in Migozzi J. (dir.), De l'écrit à l'écran : littératures populaires : mutations génériques, mutations médiatiques, Limoges, PULIM, p. 851-864.

Chalvon-Demersay, S. (2005), « Adaptations télévisuelles et figures temporelles », Réseaux, n 132, vol. 4, p. 135-184.

Chalvon-Demersay, S. (2011), "Enquête sur l'étrange nature du héros de série télévisée ", Réseaux, n 165, vol. 1, p. 181-214.

Chalvon-Demersay S. (2015), « Pour une responsabilité politique des héros de séries télévisées », Quaderni, n 88 , p. 35-51.

Esquenazi, J-P. (2010), Les séries télévisées. L'avenir du cinéma ?, Paris, Armand Colin.

Hervieu-Léger, D. (2003), Catholicisme, la fin d'un monde, Paris, Bayard.

Hughes E. (1996), « Le drame social du travail », Actes de la Recherche en Sciences Sociales, vol. 115, p. 94-99.

Jost, F. (2007), Introduction à l'analyse de la télévision, Paris, Ellipses.

Le Stang Gérard, (2008), « Séminaristes en 2008 », Études, vol. 3, Tome 408, p. 351-361.

Macé É. (2001), « “Qu'est-ce qu'une sociologie de la télévision?” (2) Esquisse d'une théorie des rapports sociaux médiatisés les trois moments de la configuration médiatique de la réalité : production, usages, représentations ", Réseaux, n 105, vol. 1 p. 199-242.

Macé É. (2002), « Sociologie de la culture de masse : avatars du social et vertigo de la méthode », Cahiers internationaux de sociologie, $\mathrm{n}^{\circ} 112$, vol. 1, p. 45-62.

Macé É. (2006), « Mouvements et contre-mouvements culturels dans la sphère publique et les médiacultures », in Maigret É. et Macé É. (dir.), Penser les médiacultures. Nouvelles pratiques et nouvelles approches de la représentation du monde, Paris, Armand Colin, p. 41-66.

Macé É., (2007), « Des “minorités visibles” aux néostéréotypes », Journal des anthropologues, Horssérie, p. 69-87.

Suaud, C. (1978), La vocation, Conversion et reconversion des prêtres ruraux, Paris, Minuit.

Verdier B. (2014), « La communication de la foi à travers le prisme médiatique d'une série télévisée : “Ainsi soient-ils”, médiateur d'un croire universel ? ", in Bratosin S. et Tudor M. (dir.), Espace Public et Communication de la foi, Montpellier, Éd. IARSIC, p. 229-241.

Verdier B. (2016), « Le discours homilétique comme dispositif de narration », Télévision, $\mathrm{n}^{\circ} 7$, p. 101-11.

Voirol O. (2005a), «Les luttes pour la visibilité : esquisse d'une problématique », Réseaux, $\mathrm{n}^{\circ}$ 129-130, p. 89-121.

Voirol O. (2005b), « Présentation. Visibilité et invisibilité : une introduction », Réseaux, n 129-130, p. 9-36. 
Willaime, J.-P. (1986), Profession Pasteur. Sociologie de la condition du clerc à la fin du XXe siècle, Genève, Labor et Fides.

Willaime, J.-P. (2008), Le retour du religieux dans la sphère publique: Vers une laïcité de reconnaissance et de dialogue, Lyon, Éd. Olivétan.

\section{NOTES}

1. Sources CEF et Beraud C. (2007) p.42-46.

2. La première saison de la série télévisée française Ainsi soient-ils a été diffusée à l'automne 2012 sur Arte, la deuxième à l'automne 2014 et la troisième à l'automne 2015.

3. Bruno Nahon, producteur de la série, dans le dossier de presse Arte, 2012.

4. «[...] d'un côté, il est le produit métamorphosé (avatar) des multiples formes spécifiques de médiations et de traductions qui concourent à sa production comme "représentation du monde "; d'un autre côté, dès lors qu'il est inscrit dans l'environnement culturel des individus et des « publics » en tant que représentation (modèle), il est, d'une manière ou d'une autre (comme nous allons le montrer), inscrit (tout comme un «modèle » dont s'inspire un peintre), dans une expérience spectatorielle, elle-même inscrite dans une expérience sociale, et pouvant donner lieu à des formes, en retour, d'actions de configuration des objets de la culture de masse et de modification des « représentations » de la réalité sociale. » (Macé, 2001, 219).

5. « Partout où des gens travaillent, il y a une différence fondamentale entre la situation de ceux qui demandent un service et la situation de ceux qui le fournissent. C'est là un aspect essentiel de ce que nous entendons par drame social du travail. » (Hughes, 1996, 95).

6. Référence de la citation, de l'intrigue dans la série. Les lettres et les chiffres entre parenthèses correspondent dans l'ordre au numéro de la saison, puis à celui de l'épisode. Dans le cas présent, il s'agit d'un extrait de l'épisode 5 de la saison 2.

7. Entretien avec les scénaristes février 2015.

8. Chaque séminaire organise dans son Pôle des Études ce genre de cours à l'extérieur du séminaire, souvent dans les universités catholiques mais pas exclusivement, en fonction de la proximité géographique.

9. Devenir Prêtre produit par KTO et réalisé par Dominique Féret en 2010 (séminaire des Carmes à Paris); Prêt(re) pour se donner réalisé par Véronique Brechot, documentaire produit par le séminaire de Lille en 2012.

10. KTO est une chaîne de télévision catholique française, fondée le 13 décembre 1999 par $\mathrm{M}^{\mathrm{gr}}$ Jean-Michel di Falco, à la demande du cardinal Jean-Marie Lustiger.

11. Billet publié le 05/10/2012 sur le blog Padreblog, tenu par des pères en majorité du diocèse de Versailles, http://www.padreblog.fr/ainsi-soient-ils-la-provoc-darte-fait-pschitt

\section{RÉSUMÉS}

Dans notre société marquée par la sécularisation, devenir prêtre au XXIe siècle interroge. Cette contribution s'intéresse à la représentation de la formation ecclésiale proposée dans la série télévisée Ainsi soient-ils diffusée sur Arte de 2012 à 2015. Nous montrons que la sémiotisation de ce dispositif de formation tend à le dé-singulariser d'une part en le replaçant sans cesse dans le 
champ d'une formation classique du supérieur et d'autre part en lui associant l'acquisition de compétences de type managérial. Le corpus est essentiellement constitué des 16 épisodes des deux premières saisons.

In our society influenced by secularization, to become a priest in the XXIth century questions. This work deals with the representation of the priesthood training through the french TV series Ainsi soient-ils broadcasted from 2012 to 2015 . We point out that the semiotization of this training setting up tends to trivialise it on the one hand because it is steadily positioned into the field of university education and on the other hand because it associates acquisition of managerial competencies. The analysis mainly concerns the sixteen episodes of the first two seasons.

\section{INDEX}

Mots-clés : représentation, formation, prêtre, série télévisée, vocation

Keywords : representation, formation, priest, TV series, vocation

\section{AUTEUR \\ BENOÎT VERDIER}

Maître de conférences en sciences de l'information et de la communication à l'Université de Reims Champagne-Ardenne, ses travaux portent sur le processus de médiatisation des collectifs de travail dans l'information, la fiction et les médias sociaux. Les groupes professionnels étudiés sont les professionnels du croire, les vignerons et les policiers. 